\title{
Tensor-Train Ranks for Matrices and Their Inverses
}

\author{
Ivan Oseledets · Eugene Tyrtyshnikov • Nickolai Zamarashkin
}

\begin{abstract}
We show that the recent tensor-train (TT) decompositions of matrices come up from its recursive Kronecker-product representations with a systematic use of common bases. The names TTM and QTT used in this case stress the relation with multilevel matrices or quantization that increases artificially the number of levels. Then we investigate how the tensor-train ranks of a matrix can be related to those of its inverse. In the case of a banded Toeplitz matrix, we prove that the tensor-train ranks of its inverse are bounded above by $1+(l+u)^{2}$, where $l$ and $u$ are the bandwidths in the lower and upper parts of the matrix without the main diagonal.
\end{abstract}

2010 Mathematical subject classification: 15A12; 65F10; 65F15.

Keywords: tensor ranks; tensor-train decomposition; QTT-ranks; inverse matrices; multilevel matrices; Toeplitz matrices; banded matrices.

\section{Introduction}

A sound idea of using the separation of variables or low-rank constructions to compactly represent a general matrix should not be applied straightforwardly, i.e. in the form of a lowrank dyadic (skeleton) decomposition of a given matrix. That cannot help if we deal with a nonsingular matrix. However, this idea works superbly when we consider Kronecker-product representations.

If $A$ is a matrix, then we denote its entries by $A(i, j)$. We write $A=G^{1} \otimes G^{2}$ when $A$ is a block matrix of the form $A=\left[G^{1}\left(i_{1}, j_{1}\right) G^{2}\right]$. As a consequence, the sizes of $A$ are products of the corresponding sizes of $G^{1}$ and $G^{2}$. In this case, $A$ is called the Kronecker product of $G^{1}$ and $G^{2}$.

Supported by the RFBR grants 11-01-00549-a, 09-01-12058, 09-01-91332 (joint with DFG), the Government Contracts П940, П1112, 14.740.11.0345 and Priority Research Grant of the Presidium and of the Department of Mathematical Sciences of the Russian Academy of Sciences. During this work the second author was a visiting professor at the University of Siedlce (Poland) and at the Hausdorff Research Institute for Mathematics in Bonn (Germany).

Ivan Oseledets

Institute of Numerical Mathematics, Russian Academy of Sciences, 8 Gubkin Street, Moscow, 119333

E-mail: ivan@bach.inm.ras.ru.

Eugene Tyrtyshnikov

Institute of Numerical Mathematics, Russian Academy of Sciences, 8 Gubkin Street, Moscow, 119333

E-mail: tee@inm.ras.ru.

Nickolai Zamarashkin

Institute of Numerical Mathematics, Russian Academy of Sciences, 8 Gubkin Street, Moscow, 119333

E-mail: kolya@bach.inm.ras.ru. 
The Kronecker product separates some variables. In order to see which variables, consider the row and column indices of $A$ as multi-indices

$$
i=i_{1} i_{1}, \quad j=j_{1} j_{2}
$$

then

$$
A\left(i_{1} i_{2}, j_{1} j_{2}\right)=G^{1}\left(i_{1}, j_{1}\right) G^{2}\left(i_{2}, j_{2}\right) .
$$

We prefer to depict multi-indices ignoring brackets and commas. The components of a mutliindex are called mode-indices. Thus, in the Kronecker product, the original variables $i$ and $j$ are not separated. Instead, we consider these indices as products of mode-indices, mix them in a special way and create new multi-indices which occur to admit separation. If we reshape $A$ into a new matrix $\tilde{A}$ so that (cf. [22])

$$
\tilde{A}\left(i_{1} j_{1}, i_{2} j_{2}\right)=A\left(i_{1} i_{2}, j_{1}, j_{2}\right),
$$

then $A$ is a Kronecker product of two matrices if and only if $\tilde{A}$ is a matrix of rank at most 1 .

It is seldom, nevertheless, that a matrix is represented by a single Kronecker product. A much wider option is when $A$ is a sum of a few Kronecker products as follows:

$$
A=\sum_{\alpha=1}^{r} G_{\alpha}^{1} \otimes G_{\alpha}^{2}
$$

$r$ is referred to as a Kronecker rank of the representation; therefore, the minimality is not required. If $r$ is the least possible value for all representations of the form (1.1), then it is called Kronecker rank of the matrix A; note the dependence on the sizes chosen for the mode-indices.

Lemma 1.1. Given decomposition (1.1), suppose that $A$ is of size $m \times n$ with $m=m_{1} m_{2}$ and $n=n_{1} n_{2}$, and $G_{\alpha}^{1}$ and $G_{\alpha}^{2}$ are $m_{1} \times n_{1}$ and $m_{2} \times n_{2}$, respectively. Then $A$ can be viewed as a block matrix

$$
A=\left[A_{i_{1} j_{1}}\right], \quad 1 \leqslant i_{1} \leqslant m_{1}, \quad 1 \leqslant j_{1} \leqslant n_{1},
$$

where the dimension of the linear span of blocks $A_{i_{1} j_{1}}$ does not exceed $r$, and is equal to $r$ if and only if $r$ is the Kronecker rank of $A$.

Proof. It is sufficient to note that each block $A_{i_{1} j_{1}}$ is an $r$-term linear combination of the blocks $G_{\alpha}^{2}$.

All the matrices on the right-hand side of (1.1) contain $r\left(m_{1} n_{1}+m_{2} n_{2}\right)$ entries. This can be notably less than $n^{2}$, the total number of entries in the matrix $A$, and thus, the ansatz of (1.1) can be useful as a compact (compressed) representation of $A$. Further compression is envisaged when we use Kronecker products with more than two factors:

$$
A=\sum_{\alpha=1}^{r} \bigotimes_{s=1}^{d} G_{\alpha}^{s} .
$$

In pursuit of better compression we are interested to take the sizes of $G_{\alpha}^{s}$ as small as possible. Hence, $d$ has to be maximized. If $m=n=2^{d}$ and all $G_{\alpha}^{s}$ are of size $2 \times 2$, then the right-hand side of (1.2) is determined by $4 r \log _{2} n$ parameters, and in the case where $r$ grows slowly as a functon of $n$, it is dramatically less than $n^{2}$. 
The actual advantage depends, of course, on $r$. Anyway, we hope to enjoy modest values of $r$ at least in some applications, and hence, we might be very interested in increasing the number of factors in the Kronecker products. Formula (1.2) defines the so called canonical polyadic (CP) decomposition of $A$. Some standard tensor decompositions and their applications have recently been surveyed in [1], the numerical calculus in higher dimensions is discussed in $[2,4]$.

A big problem with exploiting $\mathrm{CP}$ in practice is the lack of reliable and fast algorithms in the case $d \geqslant 3$. It is opposed to the case $d=2$ which is easily treated, e.g., by using the SVD. Nevertheless, one can naturally employ the idea of reducing the $d$-factor case to those with two factors. For example, this was behind the numerical examples in [21].

Consider the case $d=3$ and assume that $A$ is $m \times n$ with $m=m_{1} m_{2} m_{3}$ and $n=n_{1} n_{2} n_{3}$. The whole enterprise consists of three steps. First, we treat $A$ as a block matrix with $m_{1} \times n_{1}$ block entries and find the two-factor decomposition

$$
A=\sum_{\alpha_{1}=1}^{r_{1}} G_{\alpha_{1}}^{1} \otimes A_{\alpha_{1}}^{1}
$$

It can be found even with the least possible number of summands. Second, we find twofactor decompositions for the smaller matrices $A_{\alpha_{1}}^{1}$ which are viewed as block matrices with $m_{2} \times n_{2}$ block entries:

$$
A_{\alpha_{1}}^{1}=\sum_{\alpha_{2}=1}^{r_{2}} G_{\alpha_{1} \alpha_{2}}^{2} \otimes G_{\alpha_{1} \alpha_{2}}^{3} .
$$

Note that $r_{2}$ may depend on $\alpha_{1}$, and here we simply take the maximal value for different $\alpha_{1}$. Third, putting the former and latter together, we obtain

$$
A=\sum_{\alpha_{1}=1}^{r_{1}} \sum_{\alpha_{2}=1}^{r_{2}} G_{\alpha_{1}}^{1} \otimes G_{\alpha_{1} \alpha_{2}}^{2} \otimes G_{\alpha_{1} \alpha_{2}}^{3}
$$

In the general case, we assume that

$$
m=\prod_{s=1}^{d} m_{s}, \quad n=\prod_{s=1}^{d} n_{s}
$$

then we need $d$ steps and finish with the decomposition

$$
A=\sum_{\alpha_{1}=1}^{r_{1}} \ldots \sum_{\alpha_{d-1}=1}^{r_{d-1}} G_{\alpha_{1}}^{1} \otimes G_{\alpha_{1} \alpha_{2}}^{2} \otimes \ldots \otimes G_{\alpha_{1} \ldots \alpha_{d-1}}^{d-1} \otimes G_{\alpha_{1} \ldots \alpha_{d-1}}^{d}
$$

The result, however, does not give us permanent satisfaction: although we have obtained some CP decomposition, it contains a too large redundant total number of the involved parameters. And with increasing number of factors this redundancy tends to grow even significantly, exponentially in $d$.

A kind of remedy is still easily available. If $d=2^{p}$, then $A$ can be viewed as a block matrix with $\left(m_{1} \ldots m_{d / 2}\right) \times\left(n_{1} \ldots n_{d / 2}\right)$ block entries. Then, we begin with

$$
A=\sum_{\alpha_{1}=1}^{r_{1}} A_{\alpha_{1}}^{1} \otimes A_{\alpha_{1}}^{2}
$$


where, in the next step, $A_{\alpha_{1}}^{1}$ are treated as block matrices with $\left(m_{1} \ldots m_{d / 4}\right) \times\left(n_{1} \ldots n_{d / 4}\right)$ block entries and $A_{\alpha_{1}}^{2}$ are processed similarly as block matrices with $\left(m_{d / 2+1} \ldots m_{d / 2+d / 4}\right) \times$ $\left(n_{d / 2+1} \ldots n_{d / 2+d / 4}\right)$ block entries. Eventually we obtain some CP decomposition, where the number of summation parameters in the factors does not exceed $\log _{2} d$. If $r=\max r_{s}$, then the total number of involved parameters amounts to $r^{\log _{2} d}$; previously it was $r^{d-1}$. Note, nevertheless, that the values of $r_{s}$ are not the same as before. But, if $r$ is sufficiently small, then we may think of this new strategy as of substantial improvement, because the total number of representation parameters now grows in $d$ polynomially. Note that $r^{\log _{2} d}=d^{\log _{2} r}$.

In the next section we show how the above constructions can be modified to yield a representation with the number of parameters depending on $d$ just linearly. Strictly speaking, this is true only when the values of $r_{s}$ arising in the construction do not depend on $d$. Whatever important, the study of how these quantities behave in various situations should be postponed until the search for a potentially good ansatz is completed. As a result, we obtain the recent tensor-train representations in the case of matrices [14, 15, 16, 17, 18, 19]. A somewhat new thing is the approach: with Kronecker products we are not faced with the issue of how we should mix the indices and the cases of vectors and matrices do not differ.

Note that recursive approaches as a way to compact representations of multi-index arrays were first proposed in $[6,14,15]$. Then some algorithms with hierarchical structure were derived in [3]. However, a recursive reduction of dimensionality naturally leads to tensor trains that proved to be most convenient for designing efficient algorithms (see [16, 19]).

As soon as the tensor trains for matrices are expounded, we proceed to the study of the relations between the tensor-train ranks of matrices and their inverses. In the case of a banded Toeplitz matrix, we prove the result reported in [23]: the tensor-train ranks of the inverse are bounded above by $1+(l+u)^{2}$, where $l$ and $u$ are the bandwidths in the lower and upper parts of the matrix without the main diagonal.

\section{Tensor trains for matrices}

In the first place, we should realize that $\mathrm{CP}$ is not a must if we are only interested in better compression schemes for $A$. Nevertheless, let us elaborate on the above naive approach with some CP as a purpose (maybe a wrong one) and not refuse it too hastily.

Let us begin with $d=3$. We do not change the first step, and, as before, it yields

$$
A=\sum_{\alpha_{1}=1}^{r_{1}} G_{\alpha_{1}}^{1} \otimes A_{\alpha_{1}}^{1} .
$$

The second step, however, should be revisited. Previously the two-factor decompositions for each of the smaller matrices $A_{\alpha_{1}}^{1}$ were looked for independently:

$$
A_{\alpha_{1}}^{1}=\sum_{\alpha_{2}=1}^{r_{2}} G_{\alpha_{1} \alpha_{2}}^{2} \otimes G_{\alpha_{1} \alpha_{2}}^{3} .
$$

Now let us try to do the same simultaneously.

The matrices $A_{\alpha_{1}}^{1}$ are block matrices with $m_{2} \times n_{2}$ block entries, denote them by $A_{\alpha_{1}, i_{2} j_{2}}^{1}$. According to Lemma 1.1, for any fixed value of $\alpha_{1}$ each of these blocks is a linear combination of the blocks of $G_{\alpha_{1} \alpha_{2}}^{3}$. Consider the span

$$
\mathcal{L}=\operatorname{span}\left\{A_{\alpha_{1}, i_{2} j_{2}}^{1}, \quad 1 \leqslant \alpha_{1} \leqslant r_{1}, \quad 1 \leqslant i_{2} \leqslant m_{2}, \quad 1 \leqslant j_{2} \leqslant n_{2}\right\}
$$


of all blocks of $A_{\alpha_{1}}^{1}$ for all values of $\alpha_{1}$ and construct a basis of $\mathcal{L}$. Let it define the matrices $G_{\alpha_{2}}^{3}$, where

$$
1 \leqslant \alpha_{2} \leqslant r_{2}=\operatorname{dim} \mathcal{L}
$$

Consequently, every block $A_{\alpha_{1}, i_{2} j_{2}}^{1}$ is a linear combination of the common basis matrices $G_{\alpha_{2}}^{3}$. Denoting the coefficients by $G_{\alpha_{1} \alpha_{2}}^{2}\left(i_{2}, j_{2}\right)$, we get

$$
A_{\alpha_{1}, i_{2} j_{2}}^{1}=\sum_{\alpha_{2}=1}^{r_{2}} G_{\alpha_{1} \alpha_{2}}^{2}\left(i_{2}, j_{2}\right) G_{\alpha_{2}}^{3}
$$

Hence, by definition of the Kronecker product,

$$
A_{\alpha_{1}}^{1}=\sum_{\alpha_{2}=1}^{r_{2}} G_{\alpha_{1} \alpha_{2}}^{2} \otimes G_{\alpha_{2}}^{3}, \quad G_{\alpha_{1} \alpha_{2}}^{2}=\left[G_{\alpha_{1} \alpha_{2}}^{2}\left(i_{2} j_{2}\right)\right] .
$$

Finally,

$$
A=\sum_{\alpha_{1}=1}^{r_{1}} \sum_{\alpha_{2}=1}^{r_{2}} G_{\alpha_{1}}^{1} \otimes G_{\alpha_{1} \alpha_{2}}^{2} \otimes G_{\alpha_{2}}^{3} .
$$

We call (2.1) the tensor-train decomposition of the matrix $A$ in the case of three levels of its block partitionings. For the definition and machinery of multilevel matrices see [11, 12]. Recursive application of this approach in the case of $d$ levels gives

$$
A=\sum_{\alpha_{1}=1}^{r_{1}} \ldots \sum_{\alpha_{d-1}=1}^{r_{d-1}} G_{\alpha_{1}}^{1} \otimes G_{\alpha_{1} \alpha_{2}}^{2} \otimes \ldots \otimes G_{\alpha_{d-2} \alpha_{d-1}}^{d-1} \otimes G_{\alpha_{d-1}}^{d} .
$$

For brevity of notation it is convenient to introduce additional indices

$$
\alpha_{0}=\alpha_{d}=1
$$

and present the same decomposition in the form

$$
A=\sum_{\substack{1 \leqslant \alpha_{s} \leqslant r_{s} \\ s=1, \ldots, d-1}} \bigotimes_{k=1}^{d} G_{\alpha_{k-1} \alpha_{k}}^{k}
$$

The right-hand side of (2.2) or (2.3) is a tensor train for a d-level matrix. The quantities $r_{s}$ are called tensor-train (TT) ranks.

Note that any matrix $A$ can be viewed as a $d$-level matrix with sizes being products of smaller sizes according to (1.3): we can set $m_{s}=1$ or $n_{s}=1$ for some values of $s$. In particular, if $n=1$ then $A$ is a column matrix (vector), and its tensor-train decomposition is one of the vectors. In such a case, a $d$-level structure allows one to consider $A$ as a $d$-index array with entries $A\left(i_{1}, \ldots, i_{d}\right)$. The TT-decomposition originally defined for this case [16] looks as follows:

$$
A\left(i_{1}, \ldots, i_{d}\right)=\sum_{\alpha_{1}, \ldots, \alpha_{d-1}} \prod_{k=1}^{d} g_{k}\left(\alpha_{k-1}, i_{k}, \alpha_{k}\right) .
$$

Introducing the vectors $G_{\alpha_{k-1} \alpha_{k}}^{k}$ as ones with entries $g_{k}\left(\alpha_{k-1}, i_{k}, \alpha_{k}\right)$, we arrive at the Kroneckerproduct expression

$$
A=\sum_{\alpha_{1}, \ldots, \alpha_{d-1}} \bigotimes_{k=1}^{d} G_{\alpha_{k-1} \alpha_{k}}^{k}
$$


which exactly coincides with (2.3). A possible alternative is the matrix-product representation for the entries of $A$

$$
A\left(i_{1}, \ldots, i_{d}\right)=\prod_{k=1}^{d} M^{i_{k}}
$$

where $M^{i_{k}}$ is a matrix of size $r_{k-1} \times r_{k}$ with entries

$$
M^{i_{k}}\left(\alpha_{k-1}, \alpha_{k}\right)=g_{k}\left(\alpha_{k-1}, i_{k}, \alpha_{k}\right) .
$$

Given a $d$-level matrix $A$ with sizes subject to (1.3), we may view it as a block matrix with $\left(m_{1} \ldots m_{s}\right) \times\left(n_{1} \ldots n_{s}\right)$ block entries denoted by $A_{i_{1: s} j_{1: s}}$ with multi-indices

$$
i_{1: s}=i_{1} \ldots i_{s}, \quad j_{1: s}=j_{1} \ldots j_{s} .
$$

The blocks $A_{i_{1: s} j_{1: s}}$ are referred to as blocks of the level $s$ of $A$ [11].

Theorem 2.1. For any tensor-train representation (2.3) of a d-level matrix $A$ with sizes subject to (1.3) the TT-ranks $r_{s}$ satisfy the inequalities

$$
r_{s} \geqslant \operatorname{dim} \mathcal{L}_{s}, \quad \text { where } \mathcal{L}_{s}=\operatorname{span}\left\{A_{i_{1: s} j_{1: s}}\right\},
$$

and a tensor train exists with the equalities to be held for each $s$.

Proof. We can single out the blocks of level $s$ by rearranging the terms of (2.3) as follows:

$$
A=\sum_{\alpha_{s}=1}^{r_{s}}\left(\sum_{\alpha_{1}, \ldots, \alpha_{s-1}} \bigotimes_{k=1}^{s} G_{\alpha_{k-1} \alpha_{k}}^{k}\right)\left(\sum_{\alpha_{s+1}, \ldots, \alpha_{d-1}} \bigotimes_{k=s+1}^{d} G_{\alpha_{k-1} \alpha_{k}}^{k}\right)
$$

The inequalities for $r_{s}$ follow then directly from Lemma 1.1. They become equalities as soon as we take a common basis for the blocks of every level $s$ at each step of the recursive construction of (2.3).

In the case $m=n=2^{d}$, Theorem 2.1 was presented in [23]. Tensor-train representations in this special $d$-level case were proposed in [17] and called TTM-representations. In [17, 18] it was first disclosed that TTM can be used for amazingly efficient implementations of basic operations with those matrices and vectors that enjoy reasonably small tensor-train ranks. Eventually, using TTM may lead to a complexity logarithmic in $n$.

The case of vectors in our exposition corresponds to the values $m=2^{d}$ and $n=1$. Similar representations for vectors were advocated in [8] and called therein QTT-representations, the name emphasizing the quantization of the original size of a vector by introducing as many nontrivial mode-indices as possible. Nontrivial means attaining more than one value. Note that the very idea of increasing the number of levels artificially was proposed earlier and even somewhat studied in [21]. For various applications of QTT see $[9,10]$.

\section{TT-ranks for matrices and their inverses}

From now on we suppose that $A$ is a $d$-level matrix with $m=n=2^{d}$ and $m_{s}=n_{s}=2$ for $1 \leqslant s \leqslant d$. By TT-ranks of $A$ we mean the least possible values in all TT-representations available for $A$. The maximum of all TT-ranks for $A$ in this case will be called the QTT-rank of $A$ and denoted by $\operatorname{qttr}(A)$. 
Theorem 3.1. Assume that the a matrix $A$ is nonsingular and $\operatorname{qttr}(A)=1$. Then

$$
\operatorname{qttr}\left(A^{-1}\right)=1
$$

Proof. It suffices to note that the equation $A=U \otimes V$ and the nonsingularity of $A$ imply that $U$ and $V$ are both nonsingular and $A^{-1}=U^{-1} \otimes V^{-1}$.

Theorem 3.2. Assume that a matrix $A$ is nonsingular and $\operatorname{qtt}(A)=2$. Then

$$
\operatorname{qttr}\left(A^{-1}\right) \leqslant \sqrt{n}
$$

Proof. The claim stems from the result proved in [20]: if $A=U_{1} \otimes V_{1}+U_{2} \otimes V_{2}$, where $U_{1}, U_{2}$ are of size $p \times p$ and $V_{1}, V_{2}$ are of size $q \times q$, then $A^{-1}$ admits a similar representation with at $\operatorname{most} \min (p, q) \leqslant \sqrt{n}$ terms.

Unfortunately, the estimate of Theorem 3.2 is sharp (it follows from the considerations of [20]). To have a better estimate, we need to require from $A$ something else, e.g., some agreeable structure. Below we consider Toeplitz banded matrices.

Recall that $A$ is called a Toeplitz matrix if any its entry $A(i, j)$ depends only on $i-j$, i.e., we may set

$$
A(i, j)=a_{i-j}
$$

Bandedness means that there are nonnegative integers $l$ and $u$ such that $a_{k}=0$ whenever $k>l$ or $k<-u$. The numbers $l$ and $u$ are called the lower and upper bandwidths of $A$, respectively.

Lemma 3.1. Assume that $A$ is a Toeplitz banded matrix with lower and upper bandwidths $l$ and $u$. Then $\operatorname{qttr}(A) \leqslant 1+\lceil\sqrt{2 l}\rceil+\lceil\sqrt{2 u}\rceil$.

Proof. If $q$ is a divisor of $n$, then $A$ can be considered as a block matrix with blocks of size $q \times q$. From the structure of $A$ it emanates that there could be at most $\mu(q)=1+\lceil l / q\rceil+$ $\lceil u / q\rceil$ different blocks. Note also that these blocks are Toeplitz matrices defined by $2 q-1$ parameters. Hence, the dimension of the linear span of these blocks cannot exceed $2 q-1$. We obtain the result by maximizing $\min (\mu(q), q)$ over $q$.

Lemma 3.2. Assume that $A$ is a Toeplitz banded matrix of order $n=p q$ with lower and upper bandwidths $l$ and $u$. Then $A$ can be written as

$$
A=U_{0} \otimes V_{0}+\sum_{k=1}^{l+u} U_{k} \otimes V_{k}
$$

where $U_{k}$ and $V_{k}$ are of size $p \times p$ and $q \times q$ respectively, and additionally

$$
\operatorname{rank} V_{k}=1, \quad 1 \leqslant k \leqslant l+u \text {. }
$$

Proof. Introduce a down-shift matrix $Z_{p}$ of order $p$, it reads

$$
Z_{p}=\left[\begin{array}{cccc}
0 & & & \\
1 & 0 & & \\
& \ddots & \ddots & \\
& & 1 & 0
\end{array}\right]
$$


and let $I_{p}$ denote the identity matrix of order $p$. Evidently,

$$
A=a_{0} I+\sum_{k=1}^{l} a_{k} Z_{n}^{k}+\sum_{k=1}^{u} a_{-k}\left(Z_{n}^{\top}\right)^{k},
$$

and similarly, regarding $A$ as a block Toeplitz matrix with $p \times p$ block entries $A_{i_{1}-j_{1}}, 1 \leqslant$ $i_{1}, j_{1} \leqslant p$, we obtain

$$
A=I_{p} \otimes A_{0}+\sum_{k=1}^{\lceil l / q\rceil} Z_{p}^{k} \otimes A_{k}+\sum_{k=1}^{\lceil u / q\rceil}\left(Z_{p}^{\top}\right)^{k} \otimes A_{-k} .
$$

For illustration, let $n=8, l=3$ and $u=1$. Then

$$
A=\left[\begin{array}{cc|cc|cc|cc}
a & f & & & & & & \\
b & a & f & & & & & \\
\hline c & b & a & f & & & & \\
d & c & b & a & f & & & \\
\hline & d & c & b & a & f & & \\
& & d & c & b & a & f & \\
\hline & & & d & c & b & a & f \\
& & & & d & c & b & a
\end{array}\right]
$$

and with $p=4, q=2$ we obtain

$$
A=I_{4} \otimes\left[\begin{array}{cc}
a & f \\
b & a
\end{array}\right]+Z_{4} \otimes\left[\begin{array}{cc}
c & b \\
d & c
\end{array}\right]+Z_{4}^{2} \otimes\left[\begin{array}{ll}
0 & d \\
0 & 0
\end{array}\right]+Z_{4}^{\top} \otimes\left[\begin{array}{ll}
0 & 0 \\
f & 0
\end{array}\right] .
$$

To complete the proof, observe that the sum of ranks of the involved blocks $A_{k}, 1 \leqslant k \leqslant l$ does not exceed $l$, and the sum of ranks of $A_{-k}, 1 \leqslant k \leqslant u$ does not exceed $u$.

Lemma 3.3. [13] If a matrix of the form

$$
K=I+\sum_{i=1}^{r} A_{i} \otimes u_{i} v_{i}^{\top}
$$

with $p \times p$ matrices $A_{i}$ and column vectors $u_{i}, v_{i}$ with $q$ entries is nonsingular, then

$$
K^{-1}=I+\sum_{i=1}^{r} \sum_{j=1}^{r} A_{i j} \otimes u_{i} v_{j}^{\top}
$$

with some $p \times p$ matrices $A_{i j}$.

Theorem 3.3. Assume that $A$ is a nonsingular banded Toeplitz matrix with lower and upper bandwidths $l$ and $u$. Then $\operatorname{qttr}\left(A^{-1}\right) \leqslant 1+(l+u)^{2}$.

Proof. Let $q$ be any divisor of $n$, and set $n=p q$. By Lemma 3.2 we represent $A$ asa sum of two-factor Kronecker products (3.1). For all sufficiently small $\varepsilon>0$ it is possible to find a nonsingular $p \times p$ matrix $U_{\varepsilon}$ and a nonsingular $q \times q$ matrix $V_{\varepsilon}$ with the following properties:

(1) $\left\|U_{\varepsilon}-U_{0}\right\|_{2} \leqslant \varepsilon, \quad\left\|V_{\varepsilon}-V_{0}\right\| \leqslant \varepsilon$,

(2) $A_{\varepsilon} \equiv U_{\varepsilon} \otimes V_{\varepsilon}+\sum_{k=1}^{l+u} U_{k} \otimes V_{k}$ is a nonsingular matrix. 
Obviously, $A_{\varepsilon} \rightarrow A$ as $\varepsilon \rightarrow 0$. At the same time, for $\varepsilon>0$ we can come from $A$ to a new matrix

$$
K=A\left(U_{\varepsilon}^{-1} \otimes V_{\varepsilon}^{-1}\right),
$$

which is exactly of the form (3.3). Then we directly apply Lemma 3.3 established in [13] and remark that the Kronecker rank in the case of two-factor products cannot increase when we get to the limit.

Theorem 3.3 adds a new important feature to the already known properties of the inverses to Toeplitz banded matrices. It has been known to date that they possess a remarkable structure: the Toeplitz structure implies that the inverse is a sum of two products of Toeplitz triangular matrices, the bandedness implies that the defining parameters of those Toeplitz triangular matrices are determined through linear combinations of some vectors whose entries comprise a geometrical progression. We could have obtained some estimate of QTT-ranks by employing this structure, but we opted for a more direct approach based on Theorem 2.1.

In performing operations with matrices, we can use tensor trains as basic data structures and deal only with them during all computations. Of course, TT-ranks may grow and we need to approximate some tensor trains by other ones with smaller TT-ranks. Such an approach is viable indeed with many other data structures. In theory, we impose pretty mild requirements on these structures [5], and in practice, we surely need efficient approximation algorithms. However, it is crucial that the results of computations admit low-parametric approximations in the selected data formats. In this work, we have proved that this is so for the inversion of a Toeplitz banded matrix. Estimates for other structured matrices are the subject of on-going research.

\section{References}

[1] B. Bader and T. Kolda, Tensor decompositions and applications, SIAM Rev., 51 (2009), pp. 455-500.

[2] G. Beylkin and M. J. M. Mohlenkamp, Numerical operator calculus in higher dimensions, Proc. Nat. Acad. Sci. USA., 99 (2002), pp. 10246-10251.

[3] L. Grasedyck, Hierarchical singular value decomposition of tensors, SIAM J. Matrix Anal. Appl., 31 (2010), pp. 2029-2054.

[4] W. Hackbusch, B.N. Khoromskij, and E.E. Tyrtyshnikov, Hierarchical Kronecker tensor-product approximations, J. Numer. Math, 13 (2005), pp. 119-156.

[5] W. Hackbusch, B. N. Khoromskij, and E. Tyrtyshnikov, Approximate iterations for structured matrices, Numer. Math., 109 (2008), no. 3, pp. 365-383.

[6] W. Hackbusch and S. Kuhn, A new scheme for the tensor representation, J. Fourier Anal. Appl., 15 (2009), pp. 706-722.

[7] J. Kamm and J. G. Nagy, Optimal Kronecker Product Approximations of Block Toeplitz Matrices, SIAM J. Matrix Anal. Appl., 22 (2000), no. 1, pp. 155-172.

[8] B. N. Khoromskij, $O(d \log N)$-quantics approximation of $N-d$ tensors in high-dimensional numerical modelling, Preprint 40, MPI MIS, 2009.

[9] B. N. Khoromskij and I. V. Oseledets, DMRG+QTT approach to quantum molecular dynamics, preprint, MIS MPI, 2010.

[10] B. N. Khoromskij and I. V. Oseledets, Quantics-TT collocation approximation of parameter-dependent and stochastic elliptic PDE, Preprint 37, MIS MPI, 2010.

[11] V. Olshevsky, I. Oseledets, and E. Tyrtyshnikov, Tensor properties of multilevel Toeplitz and related matrices, Linear Algebra Appl, 412 (2006), pp. 1-21. 
[12] V. Olshevsky, I. Oseledets, and E. Tyrtyshnikov, Superfast inversion of two-level Toeplitz matrices using Newton iteration and tensor-displacement structure, Operator Theory Advances and Applications, 179 (2007), pp. 229-240.

[13] I. Oseledets, E. Tyrtyshnikov, and N. Zamarashkin, Matrix inversion cases with size-independent tensor rank estimates, Linear Algebra Appl., 431 (2009), pp. 558-570.

[14] I. Oseledets and E. Tyrtyshnikov, Recursive decomposition of multidimensional tensors, Doklady Mathematics, 80 (2009), no. 1, pp. 460-462, (In Russian: Doklady Akademii Nauk, 427 (2009), no. 1, pp. $14-16$.

[15] I. Oseledets and E. Tyrtyshnikov, Breaking the curse of dimensionality, or how to use SVD in many dimensions, SIAM J. Sci. Comput., 31 (2009), no. 5, pp. 3744-3759.

[16] I. V. Oseledets, A new tensor decomposition, Doklady Mathematics, 80 (2009), no. 1, pp. 495-496.

[17] I. V. Oseledets, On the approximation of matrices with logarithmical number of parameters, Doklady Mathematics, vol. 80, no. 2 (2009), pp. 653-654.

[18] I. V. Oseledets, Approximation of $2^{d} \times 2^{d}$ matrices using tensor decomposition, SIAM J. Matrix Anal. Appl., 31 (2010), pp. 2130-2145.

[19] I. Oseledets and E. Tyrtyshnikov, TT-cross approximation for multidimensional arrays, Linear Algebra Appl., 432 (2010), pp. 70-88.

[20] E. Tyrtyshnikov, Tensor ranks for the inversion of tensor-product binomials, J. Comput. Appl. Math., 234 (2010), no. 11, pp. 3170-3174.

[21] E. Tyrtyshnikov, Tensor approximations of matrices generated by asymptotically smooth functions, Sbornik: Mathematics, 194 (2003), no. 5-6, pp. 941-954.

[22] C. F. Van Loan and N. P. Pitsianis, Approximation with Kronecker products, NATO Adv. Sci. Ser E Appl. Sci., Kluwer: Dordrecht, 232 (1993), pp. 293-314.

[23] N. Zamarashkin, I. Oseledets, and E. Tyrtyshnikov, The tensor structure of the inverse of a banded Toeplitz matrix, Doklady Mathematics, 80 (2009), no. 2, pp. 669-670, (In Russian: Doklady Akademii Nauk, 428 (2009), no. 2, pp. 161-162). 